\title{
Quantum censorship in two dimensions
}

\author{
V. Pangon ${ }^{\mathrm{a}, \mathrm{b}}$, S. Nagy ${ }^{\mathrm{c}}$, J. Polonyi $^{\mathrm{d}, *}$, K. Sailer $^{\mathrm{c}}$ \\ a Frankfurt Institute for Advanced Studies, Universität Frankfurt, D-60438 Frankfurt am Main, Germany \\ b Gesellschaft für Schwerionenforschung mbH, Planckstr. 1, D-64291 Darmstadt, Germany \\ c Department of Theoretical Physics, University of Debrecen, Debrecen, Hungary \\ d Strasbourg University, CNRS-IPHC, BP28 67037 Strasbourg Cedex 2, France
}

\section{A R T I C L E I N F O}

\section{Article history:}

Received 8 March 2010

Received in revised form 18 June 2010

Accepted 8 September 2010

Available online 21 September 2010

Editor: J.-P. Blaizot

\section{Keywords:}

Sine-Gordon model

Renormalization group

Maxwell-cut

\begin{abstract}
A B S T R A C T
It is pointed out that increasingly attractive interactions, represented by partially concave local potential in the Lagrangian, may lead to the degeneracy of the blocked, renormalized action at the gliding cutoff scale by tree-level renormalization. A quantum counterpart of this mechanism is presented in the twodimensional sine-Gordon model. The presence of Quantum Censorship is conjectured which makes the loop contributions pile up during the renormalization and thereby realize an approximate semiclassical effect.
\end{abstract}

(C) 2010 Elsevier B.V. All rights reserved.

\section{Introduction}

A necessary condition for classical physics to emerge from quantum fluctuations is the high excitation level density or in other words, the strong degeneracy. The first condition is well known from the early days of Quantum Mechanics, the second way of expressing it implies decoherence [1,2]. This is a sharp contrast to the usual scenario of the semiclassical limit where the trajectories around the classical one are supposed to dominate the path integral. The solution of this apparent conflict arises from the remark that the dominance of the path integral by the saddle point is easy to establish for the transition amplitudes between pure states in a finite system only. Decoherence arises from degeneracy in a clear manner as soon as mixed states and density matrices are considered [3]. Another source of the degeneracy is the thermodynamical limit, the inclusion of soft modes in the system. The degeneracy of a realistic system is supposed to occur in both ways.

A large degree of degeneracy opens new problems owing to the increased quantum fluctuations. In fact, when the integrand in the path integral becomes approximately constant for infinitely many modes then the system is strongly coupled and renders our analytical methods inefficient. One may wonder whether one encouters such a scenario in the usual path integral, corresponding to either Euclidean partition function or real time transition amplitude in the thermodynamical limit. The goal of this work is to monitor the

\footnotetext{
* Corresponding author.

E-mail address: polonyi@ires.in2p3.fr (J. Polonyi).
}

degree of degeneracy by means of the functional RG method in a model where a condensate is expected.

\section{Degeneracy and functional RG method}

There have been a number of works using the functional RG method for models with spontaneously broken symmetry. For instance, the $O(N)$ symmetric scalar model was considered in Ref. [8] in $d$ dimensions for $N \geqslant 3$ where the average action, calculated in the one-loop approximation was found to be degenerate for small enough field amplitudes. But the degeneracy appears in the constrained path integral, used in this scheme as a strongly coupled dimensional model defined on the $(d-1)$-dimensional sphere in the momentum space and renders the results unreliable.

The local potential was obtained for the same model in the effective action by an approximate solution of the evolution equation [9]. The degeneracy has been identified here, too, in the unstable region. The omitted terms in the approximate expression for the potential were proportional to $\epsilon_{d e g}$, the extent of the nondegeneracy of the action in the unstable region. The problem with this result is that the small parameter of the loop expansion, used to derive the evolution equation of the average potential is $\mathcal{O}\left(\epsilon_{\text {deg }}^{-1}\right)$ rendering the uncertainty of the solution $\mathcal{O}\left(\epsilon_{\text {deg }}^{0}\right)$. The ansatz for the effective action has been improved in Ref. [10] by including the wave function renormalization constant but the local potential was reduced to a simple quartic polynomial and such a truncation prevents us to address the issue of degeneracy. The limit of a large number of fields offers exact solutions and the effective action was shown to be degenerate in this limit [11]. But as soon as 
the inverse of the number of fields becomes comparable with some dimensionless measure of degeneracy then this limit becomes misleading from the point of view of our problem. In addition, the Higgs mode was totally ignored in favor of the Goldstone modes in the argument, an unreliable approximation when a possible deviation from an infinitely degenerate situation is sought.

Another attempt to see the impact of the degeneracy on the dynamic was based on the use of sharp cut-off. The tree-level evolution of the local potential of the blocked bare action was followed in Ref. [4]. The saddle point for the blocking when the cut-off is lowered as $k \rightarrow k-\Delta k$ is trivial for large enough $k$. For smaller $k$ the action develops non-trivial minima for fluctuations at the gliding cut-off and inhomogeneous saddle points appear. The result is a dynamical Maxwell-cut, the degeneracy of the bare action for small wave numbers and small values of the condensate. This method has two weak points. One is that one cannot include wave function renormalization constant when sharp cutoff is used. The other is that the variation of the bare action for the first appearance of the instability is $\epsilon_{d e g}=\mathcal{O}(\Delta k / k)$ and the small parameter of the expansion where the loop-integral is carried out on a shell of thickness $\Delta k$ in the momentum space is $\mathcal{O}(\Delta k / k) / \epsilon_{\text {deg }}=\mathcal{O}\left((\Delta k / k)^{0}\right)$.

When dealing with the average or the bare action we have to rely on the loop-expansion eventhough the approximate or exact solution of the evolution equation resums partially or completely the higher loop contributions. We have seen that the degeneracy arising with the condensate renders these approaches unreliable. No loop expansion is evoked in deriving the evolution equation of the effective action in Refs. [12,11,13] and one would hope that this method remains applicable for condensates. But the truncation of the evolution equation brings the problem back. The effective action is convex by definition and the resulting Maxwellcut hides the dominant physics, related to domain walls as soft, non-perturbative modes behind a degenerate effective action functional. The evolution is described by an autonomous equation thus the information about the dynamics of the unstable, degenerate region must be provided by the way it is joined to the stable, nondegenerate part of the dynamics. The non-analyticity prevents us to use the traditional Landau-Ginzburg double expansion strategy and we have no guidance left to perform the unavoidable truncation of the functional differential equation in this region. The generator functional, or the effective action in particular is always used in Quantum Field Theory as a book-keeping device to manipulate hierarchical equations for different Green-functions. No convergence or existence issues are raised in this manner. But as soon as the functional is born by solving non-linear equations rather than assuming the availability of functional Taylor series we face a radically more difficult mathematical issue, reminiscent of the bound state problem.

There is another problem when the evolution of the effective action is considered in the presence of condensate. The effective action approaches a singular limit, set by the Maxwell-cut in a manner which is determined by the suppression term introduced in this scheme. This latter stabilizes the dynamics and provides the analytical continuation to derive an evolution equation in the vicinity of a singularity. It seems that the singularity is avoided for sufficiently strongly scale dependent suppressions [14] indicating that the analytical continuation provided by the arbitrary suppression term may not be unique and artificial fixed points might be generated at the edge of the non-convex region. This problem is absent for sharp cut-off where each mode is taken into account with its original dynamics and no analytical continuation is made.

We make a step forward in this work by increasing the numerical flexibility of the functionals followed in the evolution for the two-dimensional sine-Gordon model. We still rely on the gra- dient expansion but renounce the expansion in the field amplitude and allow arbitrary field dependence during the evolution in the hope that such a richer ansatz allows us to reach better the formation of the condensate. We shall consider the evolution of the bare action given by the Wegner-Houghton equation during the lowering of a sharp cut-off. It is found for certain values of the coupling constants that the quantum fluctuations prevent the system from developing a degeneracy despite the emergence of the Maxwell-cut in the effective potential. What is new in this result is that an almost degenerate, regular dynamics is now established without relying on the non-unique, regulating effects of an arbitrary suppression mechanism. Guided by an analogous problem in General Relativity such a quantum fluctuation generated way to reproduce the salient feature of the semiclassical physics without saddle points might be called the Quantum Censorship (QC) in Quantum Field Theory [7]. QC may not be established in some other part of the coupling constant space because one cannot discover a degeneracy by means of a numerical method of finite accuracy. Analytical methods are even less useful for such a degenerate dynamics. Therefore, the reliable clarification of the presence or absence of QC remains to be an open problem.

\section{Saddle points of the RG equations}

We start by inspecting the way the renormalization group method, the most promising non-perturbative tool in quantum field theory, indicates the presence of condensate in the vacuum. The question one wants to clarify is the scale where the condensate shows up first as a singularity in the renormalized dynamics as the cutoff of the theory is lowered.

The renormalized trajectory of a quantum system maps out the scale dependence of the effective parameters of the system. It is one of the basic tenets of the renormalization group procedure that the critical behaviors, the singular dependence of the IR observables on the UV parameters, builds up by scanning through infinitely wide scale regions rather by a singularity observed at a finite scale. Correspondingly, the renormalized trajectory should at least be continuous in the scale parameter, an expectation which has already been justified for local quantum field theories [5]. But the continuity of the renormalized trajectory in the cutoff does not exclude other singularities.

Let us consider a Euclidean theory characterized by the action $S_{k}[\phi], k$ being the sharp UV cutoff, and write the field variable as $\phi+\phi^{\prime}$ where the supports of $\phi$ and $\phi^{\prime}$ in Fourier space are $|p|<k-\Delta k$ and $k-\Delta k<|p|<k$, respectively. An infinitesimal blocking step corresponds to integrating out the modes close to the cutoff, giving the functional integration

$e^{-\frac{1}{\hbar} S_{k-\Delta k}[\phi]}=\int D\left[\phi^{\prime}\right] e^{-\frac{1}{\hbar} S_{k}\left[\phi+\phi^{\prime}\right]}$

which may possess a saddle point. The derivatives of the trajectory with respect to the cutoff are obviously singular at the scale where this saddle point appears or disappears. Such a tree-level renormalization has been found in the spinodal instability (SI) regions and was responsible for the degeneracy of the blocked action at the cutoff scale for certain homogeneous background field $\phi$ in the $\phi^{4}$ and the sine-Gordon (SG) model [4,6], a dynamical generalization of the Maxwell-cut.

The blocking (1) yields a functional finite difference equation whose solution lies well beyond our analytical capabilities. It is usually handled by imposing rather simple restrictions, either by ignoring altogether the loop contributions to the blocking or restricting the evolution of the action into few coupling constants. In the context of the SG model one retains some Fourier coefficients of $V_{k}(\phi)$ in the local potential approximation [6], 
$S_{k}[\phi]=\int_{x}\left[\frac{1}{2}\left(\partial \phi_{x}\right)^{2}+V_{k}\left(\phi_{x}\right)\right]$.

The saddle points considered were plane waves and the degeneracy of the action for modes at the cutoff was found by recovering the potential $V_{k}^{S I}(\phi)=-k^{2} \phi^{2} / 2$ in the SI region, in a certain interval for $\phi$. The inhomogeneous saddle points generate non-perturbative soft modes, the zero modes corresponding to the breakdown of the external, space-time symmetries. Beyond these intervals for the field the theory appeared to be stable, without unexpected soft modes. At the end of the intervals, at the border of the stable and unstable regions the loop corrections make the potential non-analytical [4] and the truncation of the potential, behind any expansion scheme, becomes highly suspicious.

Beyond the problem of justifying the omission of the loop correction or the possible non-analytical structures in the potential there is an even more fundamental issue here. The finite difference equation (1) contains infinitely many higher loop contributions which are suppressed by the small parameter $\epsilon_{k}=\hbar \Delta k / k\left|\ln \lambda_{\min }\right|$, $\lambda_{\min }$ being the smallest eigenvalue of $\delta^{2} S[\phi] / \delta \phi \delta \phi$. The availability of the loop expansion is assumed in deriving the evolution equation which in turn resums the expansion in the differential equation limit, $\Delta k / k \rightarrow 0$. There are two ways $\epsilon_{k}$ can become large, either for $k \rightarrow 0$ or for $\lambda_{\text {min }} \rightarrow 0$. The first possibility, a singular thermodynamical limit, is discarded for the usual, local models. The second alternative, the case of degenerate action is more realistic. Once the blocked action becomes exactly degenerate at the cutoff scale where it is supposed to describe best the dynamics then the integral in (1) obeys no expansion anymore and we have no analytical tool left to tackle the problem. Therefore, certain singularities of the renormalized trajectory, such as the degeneracy of the action may have serious consequences.

\section{SG model}

The theory considered in this work is defined by the action of (2) in two-dimensional Euclidean space-time where the bare potential at the initial cutoff, $k_{\text {init }}=\Lambda=1$, is $V_{B}(\Phi)=$ $k^{2} \tilde{u}_{B} \cos \left(\sqrt{8 \pi} \beta_{r} \Phi\right)$. It exhibits a $Z_{2}$ symmetry $\phi(x) \rightarrow-\phi(x)$ and periodicity in the internal space, $\phi(x) \rightarrow \phi(x)+2 \pi / \beta(\beta=$ $\left.\sqrt{8 \pi} \beta_{r}\right)$. The evolution of the local potential is governed by the Wegner-Houghton equation [15] in $d=2$

$\left(2+k \partial_{k}\right) \tilde{V}_{k}(\phi)=-\frac{1}{4 \pi} \ln \left(1+\tilde{V}_{k}^{\prime \prime}(\phi)\right)$,

in terms of the dimensionless potential $\tilde{V}_{k}=k^{-2} V_{k}$, in the absence of the non-trivial saddle point in (1). Note that the argument of the logarithm function is the restoring force acting on the quantum fluctuations at the cutoff and should be positive to justify the loop expansion. When the argument becomes negative then this equation is not valid anymore and the saddle point contributions to the integral of (1) have to be taken in account.

The model is known to exhibit two phases $[16,17,6,18]$, separated by the Coleman point, $\beta_{r}=1$. The simplest indication is the change of the sign of the one-loop beta-function for $\tilde{u}$ at this point, $\tilde{u}_{k} \approx \tilde{u}_{B}\left(k^{2} / \Lambda^{2}\right)^{\beta_{r}^{2}-1}$. The phase $\beta_{r}>1$ or $\beta_{r}<1$ preserves or breaks the internal symmetries, respectively. The phase with broken symmetry is equivalent with the neutral sector of the massive Thirring model [16] and the neutral Coulomb-gas [19]. The lattice regulated SG model can be mapped to the planar XY model [20] providing a non-perturbative RG flow.

The symmetry broken phase contains further special points. Higher, $n$-th order perturbative contributions generate the potential $u_{n} \cos \left(\sqrt{8 \pi} \beta_{r} n \Phi\right)$ and the corresponding coupling strength is renormalizable or non-renormalizable for $\beta_{r}<\beta_{r}^{(n)}=1 / n$ or $\beta_{r}>\beta_{r}^{(n)}$, respectively. Therefore, the theory with $\beta_{r}^{(n+1)}<$ $\beta_{r} \leqslant \beta_{r}^{(n)}$ has $n$ perturbatively renormalizable parameters apart of $\beta$. The UV scaling regime was found to be very limited due to intermediate scaling laws, appearing in between the UV and the approximate SI scaling regimes where all coupling constants grow with decreasing cutoff [18]. The point $\beta_{r}^{(4)}$ deserves special attention. The duality established in the Villain model [21], $(\beta, u, z) \rightarrow(2 \pi / \beta, 2 z, u / 2)$ where $z$ denotes the vortex fugacity, maps the XY model without external field, $u=0$, into the continuum SG model [20]. A special feature of the continuum SG model is its non-periodic kinetic energy which suppresses the vortices in the $\mathrm{XY}$ model context, configurations with point singularity. Thus the $z=0$ plane corresponds to the continuum SG model, studied in this work. The dual of the Coleman-point $\beta_{r}=1$ is the KosterlitzThouless critical point, $\beta_{r}^{(4)}$.

The SG model is similar to non-Abelian gauge theories in what the field variable is compact. The effective potential of a compact variable is flat, the constant being the only function which is periodic and convex in the same time. Therefore, the effective potential, $V_{k=0}(\Phi)$ cannot distinguish the phases and one expects similar phenomena in non-Abelian gauge theories, as well. It was found that the potential $\tilde{V}_{k}(\Phi)$, expressed in units of the cutoff, solves this problem and can be used to identify the phase structure [6].

It is natural to represent the periodic potential of the SG model by a Fourier series. But the Fourier series of the potential $V_{k}^{S I}(\phi)=$ $-k^{2} \phi^{2} / 2$ for $-\pi / \beta<\phi<\pi / \beta$ with its periodic extension seen approximately in the IR region by following the evolution of a truncated Fourier series [18], converges badly and all we can ascertain is that an approximate degeneracy occurs in the symmetry broken phase. Note that the Fourier-expansion based numerical solution of different evolution equations $[22,12,13,23]$ suffers the same problem and it is difficult to decide whether the SI occurs or not.

We avoid the limitations of a truncated series by solving the evolution equation for unconstrained potential numerically. The potential is represented in the algorithm by a spline i.e. a piecewise Chebyshev polynomial [7]. The evolution of the coefficients of the Chebyshev polynomials are followed in this method and the linear algebra employed becomes singular for degenerate actions. The internal consistency checks of the algorithm, controlling the derivatives and the integration adjust the step size, $\Delta k$ dynamically and stops the execution of the computer program when the partial error in the algorithm reaches the precision of the number representation in the computer.

\section{Coleman point}

The theory with $\beta_{r}=1$ separates two phases [16]. The RG flow in the $\beta_{r}>1$ phase which is usually referred to as the nonrenormalizable phase gives a simple evolution due to the smallness of $\tilde{u}$. What is more interesting for us is that the potential barrier between two neighboring minima is thinner for large $\beta_{r}$ and the fluctuations can "fill up" the minima easier. The result is QC, the stable, loop-generated and gradual approach of the potential $V_{k}^{S I}(\phi)$ as the cutoff is lowered with the establishment of exactly degenerate action for $k \rightarrow 0$ only. This scenario was actually established in this phase by following the evolution of the local potential, represented by a truncated Fourier expansion [18,24]. The small $\beta_{r}$, or renormalizable phase shows an interesting, more involved structure because the barrier between the minima of the potential is wider and $\mathrm{QC}$ is more difficult to realize. The approximation $[18,24]$ indeed leads here to degenerate action and 


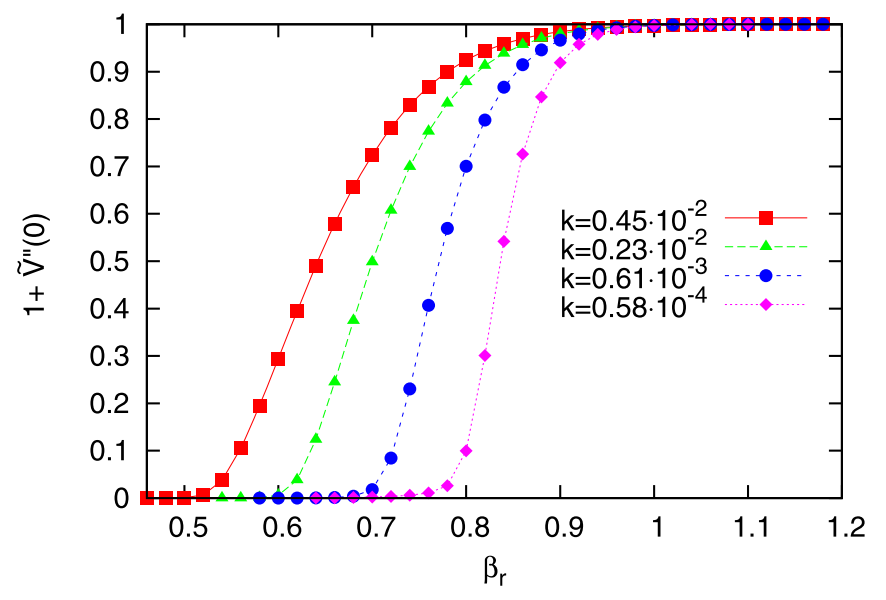

Fig. 1. The second derivative of the action is plotted as the function of the relative parameter $\beta_{r}$, for various values of the scale $k$. It goes to approximately zero when $\beta_{r}<1$ and to 1 when $\beta_{r}>1$.

to tree-level renormalization and QC is prevented to act. The result is a super-universal, strictly bare parameter independent shape for the local potential within the unstable region.

The numerical solution of the evolution equation with unconstrained potential confirms the flattening of the dimensionless potential for $\beta_{r}>1$, as one can see in Fig. 1 where $1+\tilde{V}^{\prime \prime}(\phi=0)$ is plotted for different values of the cutoff $k$. What we see in the symmetry broken phase, $\beta_{r}<1$, is that the action is nearly degenerate, $1+\tilde{V}^{\prime \prime}(0) \approx 0$, the argument of the logarithm function in (3) is nearly vanishing and the evolution is very close to be singular.

\section{Descent in $\beta$}

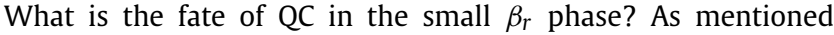
above, neither analytical nor numerical methods are available to our knowledge to answer this question. No analytical method is known to regulate and to handle functional integrals with constant integrands. Even if we grant the evolution equation (3), no numerical algorithm, realized by finite computing power can distinguish exact degeneracy from small but finite variation. What is left is to collect circumstantial evidences to support our conjecture, spelled out below.

We start on the analytical side, by noting that the almost degeneracy of the action at the cutoff as shown in Fig. 1 generates large amplitude, non-perturbative modes with small but finite wave numbers, the hallmark of spontaneous symmetry breaking. It is the fundamental group symmetry which might break at this point, the invariance of the theory with respect to the shift $\phi(x) \rightarrow \phi(x)+2 \pi / \beta$. The breakdown of this symmetry is realized by restricting the functional integral over field configurations with a given number of topological charge. In fact, the derivation of the correct Schwinger-Dyson hierarchical equations, obtained formally by performing infinitesimal variation of the field variables, requires to integrate in the path integral over a field-configuration space which is closed under smooth deformations. The smallest domain satisfying this condition consists of a single homotopy class, describing the propagation of a fixed number of kinks. The spontaneous breakdown of the fundamental group symmetry is realized by the dynamical restriction of the integration domain of the path integral for infinitely large systems. The SG model is asymptotically free in the UV limit in the symmetry broken phase and its semiclassical solution reveals stable kinks. The important message of this line of thought from our point of view is that the dynamical stability of kinks lends stability for certain inhomoge-

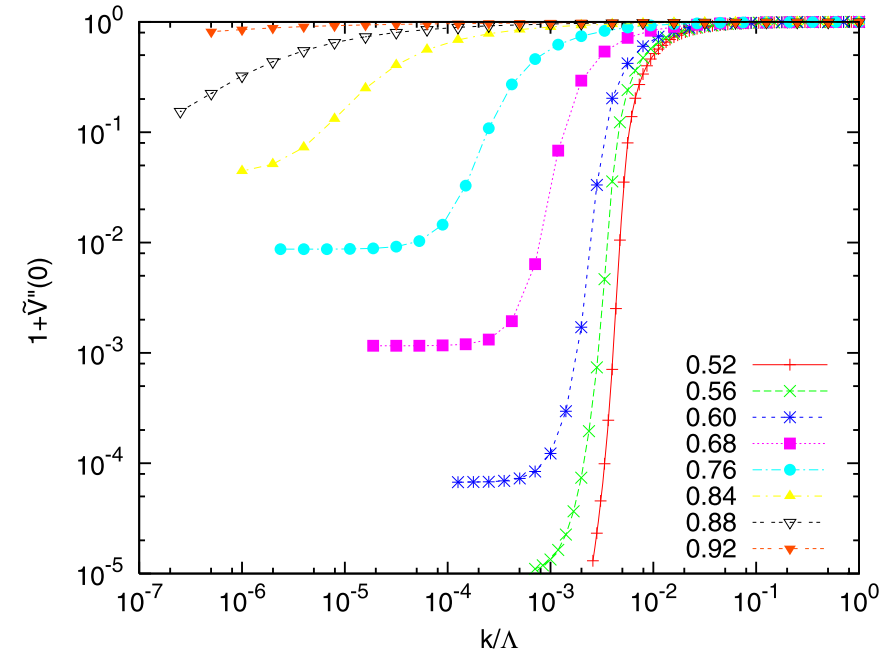

Fig. 2. The saturation of the curvature at small $k$ for various values of $\beta_{r}$. The values of $\beta_{r}$ are shown for each curve.

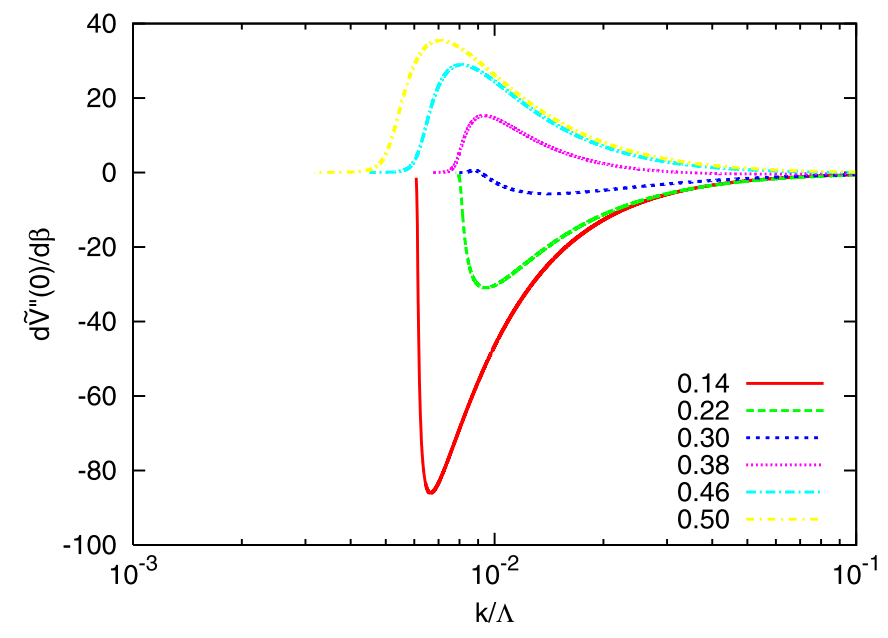

Fig. 3. The sensitivity matrix element up to a sign, $S_{0}$, as the function of the scale for various $\beta_{r}$. The values of $\beta_{r}$ are shown for each curve.

neous configurations in the blocking, (1) and thereby may prevent QC to be realized. The numerical results confirm this tendency. The scale dependence of the degeneracy $1+\tilde{V}^{\prime \prime}(0)$, depicted in Fig. 2, indicates that the loop corrections renormalize the action to a non-degenerate, scale invariant form below a crossover scale which moves in the IR direction as we penetrate into the symmetry broken phase. This result, not foreseen in the previous RG calculations, requires an unconstrained treatment of the local potential. The horizontal segments in Fig. 2 signal that QC operates for $\beta_{r}^{(2)}<\beta_{r}<\beta_{r}^{(1)}$ but with a strength which decreases with $\beta_{r}$. The decrease of $\mathrm{QC}$ forces $1+\tilde{V}^{\prime \prime}(0)$ to drop earlier during the evolution which increases the scale windows of the almost degenerate action. For $\beta_{r}<\beta_{r}^{(2)}$ either the trajectory is stabilized at a degeneracy level which is not detectable within the accuracy of the double precision number representation or the steep drop of the degeneracy continues until we hit true degeneracy and eventually generate saddle points. The degree of degeneracy of the action can be further explored by means of the sensitivity matrix $\mathcal{S}_{k}$ whose elements are the derivatives of the renormalized quantity $\tilde{V}_{k}^{\prime \prime}(0)$ with respect to the bare parameters, in particular $\mathcal{S}_{k}=\partial \tilde{V}_{k}^{\prime \prime}(0) / \partial \beta_{r}$ in the present case which is shown in Fig. 3. For approximately $\beta_{r}>\beta^{(4)}$ it starts with negative values at the cutoff 


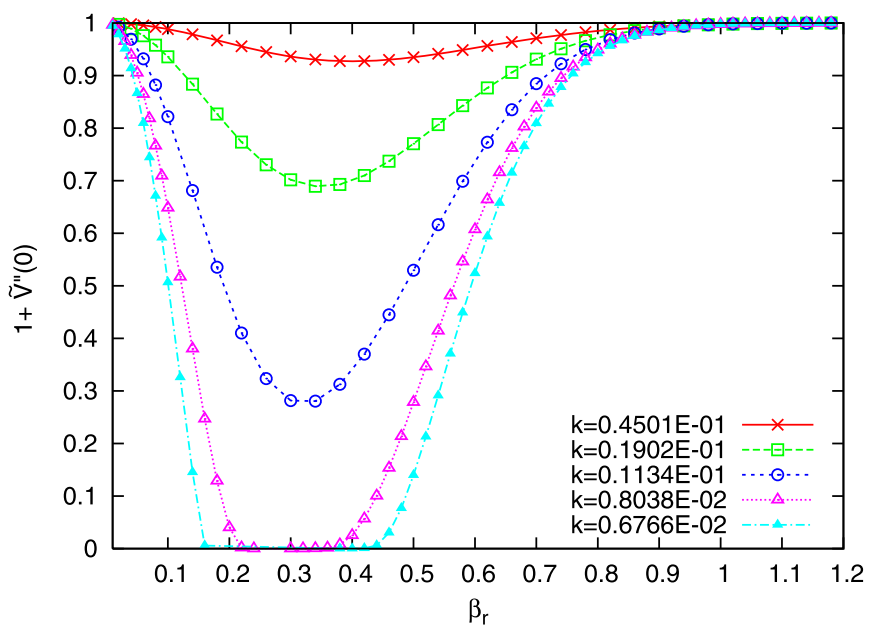

Fig. 4. The curvature in $\phi=0$ of the action as the function of $\beta_{r}$ for various scales. It displays maximal speed for the flow at $\beta_{r} \sim \beta_{r}^{(3)}$.

(not visible in the figure) but traverses zero and becomes positive at a scale which moves in the UV direction as $\beta_{r}$ is raised. For $\beta^{(3)}<\beta_{r}<\beta^{(2)}$ the positive peak is higher and the negative one is lower than one. For $\beta^{(4)}<\beta_{r}<\beta^{(3)}$ the two peaks have comparable heights. There is a separatrix in the renormalization group flow at $\beta_{r} \sim \beta_{r}^{(3)}$ reflecting a competition between Coleman and Gaussian FP, cf. Fig. 4. Finally, for approximately $\beta_{r}<\beta^{(4)} \mathcal{S}_{k}$ stays negative and develops a strong peak. Such a dependence is consistent with a strengthening degeneracy as $\beta_{r}$ is decreased between $\beta^{(2)}$ and $\beta^{(3)}$. The further decrease of $\beta_{r}$ seems to increase the degeneracy even more. The dominant, IR part of the matrix element obeys the approximate scaling law $\mathcal{S}_{k} \approx \pm 1 / k^{2}$ with a high accuracy until the evolution changes abruptly for $\beta_{r}<\beta^{(4)}$. It is far from clear if this is a precursor of a turn towards degeneracy.

Note that the appearance of the special values $\beta_{r}^{(n)}$ in the features discussed above is natural, these are the $\beta_{r}$ values where the UV critical exponent of a coupling constant changes sign, altering in a profound way the competition between the various Fourier modes in approaching the degenerate action.

\section{Summary}

It is pointed out in this Letter that characteristically classical dynamics, such as the classical collective coordinate generated Maxwell-cut, might be mimicked by quantum fluctuations with a surprising accuracy. Such a smearing of the usual singularities of the tree-level contributions is called QC.

The thumb rule to estimate the strength of $\mathrm{QC}$ is to find the strength of fluctuations which may have similar effects than the classical saddle points. Note that the functional integration in question, the blocking (1), is over a UV subspace of configurations $\phi^{\prime}$ only and the IR background field $\phi$ can stabilize inhomogeneous saddle points even if the full functional integral of the theory possesses no such saddle points. The fluctuations around the saddle points are strong in general if the action has a shallow minimum at the saddle point. The fluctuations which may wash different saddle points together are strong if different saddle points are close in the field space. The distinguished feature of the SG model is the periodicity of its potential which allows us to control the latter type of fluctuations by the parameter $\beta_{r}$ and makes this model a good testing ground for QC.

Circumstantial evidences were presented for the gradual weakening QC in the two-dimensional SG model as the period length of the potential is increased in the field space. But the final word about the fate of $\mathrm{QC}$ in the small $\beta_{r}$ part of the phase diagram remains a provocative open problem.

We have considered vacuum expectation values in this work from the point of view of QC. Another issue what remains open whether the full classical behavior, decoherence included can be reproduced by QC. We plan to return to this problem in a future publication.

\section{Acknowledgements}

S. Nagy, J. Polonyi and K. Sailer acknowledge an MTA-CNRS grant.

\section{References}

[1] H.D. Zeh, Found. Phys. 1 (1970) 69.

[2] W.H. Zurek, Phys. Rev. D 24 (1981) 1516.

[3] J. Polonyi, Phys. Rev. D 74 (2006) 065014.

[4] J. Alexandre, V. Branchina, J. Polonyi, Phys. Lett. B 445 (1999) 153.

[5] R.B. Israel, in: J. Fritz, J.L. Lebowitz, D. Szász (Eds.), Random Fields, Esztergom, 1979, North-Holland, Amsterdam, 1981;

A.C.D. van Enter, R. Fernández, A. Sokal, J. Stat. Phys. 72 (1993) 879.

[6] I. Nándori, J. Polonyi, K. Sailer, Phys. Rev. D 63 (2001) 045022.

[7] V. Pangon, S. Nagy, J. Polonyi, K. Sailer, arXiv:0907.0144, Phys. Rev., submitted for publication.

[8] A. Ringwald, C. Wetterich, Nucl. Phys. B 334 (1990) 506.

[9] N. Tetradis, C. Wetterich, Nucl. Phys. B 383 (1992) 197.

[10] C. Wetterich, Z. Phys. C 57 (1993) 451.

[11] J. Berges, N. Tetradis, C. Wetterich, Phys. Rep. 363 (2002) 223.

[12] T.R. Morris, Internat. J. Modern Phys. A 9 (1994) 2411;

M.E. Fisher, Rev. Modern Phys. 70 (1998) 653; C. Bagnuls, C. Bervillier, Phys. Rep. 348 (2001) 91.

[13] J. Polonyi, Central Eur. J. Phys. 1 (2003) 1.

[14] D. Litim, J. Pawlowski, L. Vergara, Convexity of the effective action from functional flows, arXiv:hep-th/0602140.

[15] F.J. Wegner, A. Houghton, Phys. Rev. A 8 (1973) 401.

[16] S.R. Coleman, Phys. Rev. D 11 (1975) 2088.

[17] S. Mandelstam, Phys. Rev. D 11 (1975) 3026; B. Schroer, T. Truong, Phys. Rev. D 15 (1977) 1684; D.J. Amit, Y.Y. Goldschmidt, G. Grinstein, J. Phys. A 13 (1980) 585; A.B. Zamolodchikov, Internat. J. Modern Phys. A 10 (1995) 1125;

J. Zinn-Justin, Quantum Field Theory and Critical Phenomena, Clarendon Press, Oxford, 1996.

[18] S. Nagy, I. Nándori, J. Polonyi, K. Sailer, Phys. Lett. B 647 (2007) 152; S. Nagy, I. Nándori, J. Polonyi, K. Sailer, Phys. Rev. Lett. 102 (2009) 241603.

[19] S. Samuel, Phys. Rev. D 18 (1978) 1916.

[20] K. Huang, J. Polonyi, Internat. J. Modern Phys. 6 (1991) 409.

[21] J.V. José, L.P. Kadanoff, S. Kirkpatrick, D.R. Nelson, Phys. Rev. B 16 (1977) 1217.

[22] J. Polchinski, Nucl. Phys. B 231 (1984) 269.

[23] J. Pawlowski, Ann. Phys. 322 (2007) 2831.

[24] S. Nagy, I. Nándori, J. Polonyi, K. Sailer, Phys. Rev. D 77 (2008) 025026. 